\title{
Quality and safety in abdominal imaging: How important?
}

\author{
David M. Paushter
}

Department of Radiology, The University of Chicago, 5841 S. Maryland Ave., MC 2026, Chicago, IL 60637, USA

In this issue of Abdominal Radiology, there is a series of articles covering multiple facets of quality and safety. Although the majority of information presented is applicable to all aspects of radiology, abdominal imaging provides a unique set of circumstances due to its utilization of a large number of modalities, inclusion of invasive procedures, need for integration with a broad swath of institutional and interdepartmental imperatives, and potential for harm. In their article, Imaging Wisely, $\mathrm{Lu}$ and Thomas [1] provide insight and guidelines for the appropriate and safe use of $\mathrm{CT}$ to limit patient radiation. In addition to scanner-based CT dose reduction techniques, the importance of selecting the most appropriate modality and CT protocol for the clinical indication is highlighted. As healthcare moves toward efficiency, quality and low cost of services, the key position of the radiologist is highlighted.

The article on MR safety by Sammet [2] not only provides a primer on MR physics, but provides practical guidance for patient safety as well as the current status of regulation. Although there is heightened awareness of the potential dangers of ferromagnetic objects in magnetic fields and malfunction of devices such as pacemakers, other tangible risks, such as burns and hearing loss, exist, as well as exam limiters and patient dissatisfiers associated with claustrophobia. In our article, Paushter and Thomas [3] have attempted to provide an overview of quality improvement techniques with examples from our specialty. Although some have been leery of the application of industrial techniques to healthcare, this transition has been successful and is well underway. Application of even the most basic quality improvement principles and methodology provides a framework for problem solving and goal attainment.

Correspondence to: David M. Paushter; email: dpaushter@uchicago.edu
Kruskal et al. [4] provide a critical look at the current status of peer review in abdominal imaging, with a clear need to move away from potential punitive reporting of discrepancies to meet external regulations, towards group and individual learning to improve outcomes. The authors provide methodology to improve the review process and allow meaningful performance improvement. Sahni and Khorasani [5] address the critical role of results reporting in patient care. Accurate and appropriate communication is required at every step of the imaging study, including ordering, appropriateness evaluation, protocoling, and "actionable" reporting. The timely topic of integration of evidence-based computer decision support is discussed in detail, including obstacles to successful implementation and limitations of supportive evidence-based improvement in outcomes. Issues of efficiency and standardization are addressed within a framework of growing electronic tools that assist the radiologist in communication.

Berlin and Duszak [6] frame the "value equation" within the evolving framework of new healthcare systems, with emphasis on the expanding role of the radiologist in standardization, utilization management, and patient safety. Not only is quality improvement the "right thing to do", but in economic terms is a key to reimbursement through efficiency and improved patient outcomes. As our field continues to evolve, proven methods of quality improvement will need to be further internalized in our workflow, and data accrued on patient outcomes and overall impact on healthcare. The historic development and implementation of efficiency tools to improve the working environment for radiologists must be replaced with a value-based view of efficiency and its ultimate effect on the patient. This requires intimate involvement of the radiologist in quality improvement as an integral component of health care systems, and as physicians with a primary goal of excellence in patient care. 


\section{References}

1. Lu ZF, Thomas S (2016) Imaging wisely: patient safety in CT. Abdom Radiol. doi:10.1007/s00261-016-0676-0

2. Sammet S (2016) Magnetic resonance safety. Abdom Radiol. doi: 10.1007/s00261-016-0680-4

3. Paushter DM, Thomas S (2016) Quality assurance methodology and applications to abdominal imaging PQI. Abdom Radiol. doi: $10.1007 / \mathrm{s} 00261-016-0678-\mathrm{y}$
4. Kruskal JB, Eisenberg RL, Brook O, Siewert B (2016) Transitioning from peer review to peer learning for abdominal radiologists. Abdom Radiol. doi:10.1007/s00261-016-0675-1

5. Sahni VA, Khorsani R (2016) The actionable imaging report. Abdom Radiol. doi:10.1007/s00261-016-0679-x

6. Berlin JW, Duszak R (2016) The role of the radiologist in new payment systems. Abdom Radiol. doi:10.1007/s00261-016-0674-2 